\title{
Uterine arteriovenous malformation with repeated vaginal bleeding after dilatation and curettage
}

\author{
Da Joung Shim, Sang Joon Choi, Ji Min Jung, Ji Hyun Choi \\ Department of Obstetrics and Gynecology, Chosun University Hospital, Chosun University College of Medicine, Gwangju, Korea
}

Uterine arteriovenous vascular malformation (UAVM) is a disease that causes excessive bleeding. The symptoms do not subside without proper treatment and this can lead to life-threatening situations. The correct diagnosis of UAVM can be complicated if the patient's uterus did not completely discharge everything during abortion (in broader terms, retaining remnants of the products of conception). In this case, Doppler ultrasonography and computed tomography angiography with 3-dimensional rendering were used to analyze the cause of bleeding and provide proper treatment of this patient. Then, uterine artery embolization, dilatation, and curettage were performed safely and successfully. The patient no longer had symptoms of vaginal spotting during the planned follow up care. UAVM is uncommon; however, if reproductive-age women show repeated abnormal vaginal bleeding after dilatation and curettage, a diagnosis of UAVM must be considered based on the medical history and examination.

Keywords: Arteriovenous malformation; Uterine artery; Vaginal bleeding; Ultrasonography

\section{Introduction}

Uterine arteriovenous vascular malformation (UAVM) is a less commonly known condition that is potentially lifethreatening due to excessive bleeding and results from an abnormal communication between arteries and veins of the uterus. UAVM is typically classified as congenital or acquired. This case report describes an acquired UAVM in a 37-yearold woman who experienced repeated episodes of vaginal bleeding after undergoing dilation and evacuation due to a missed abortion at 9 weeks of gestation. Upon her visit to our hospital, the Doppler ultrasonography result indicated increased vascularity and prominent vessels in the fundus of the uterus. But the patient also had retained products of conception, making the diagnosis complicated. UAVM was confirmed on computed tomography (CT) angiography with 3-dimensional (3D) rendering. Uterine artery embolization (UAE), dilatation, and curettage were performed safely and successfully.

\section{Case report}

A 37-year-old nulligravida was referred to our hospital for repeated episodes of vaginal bleeding for 10 days after dilation and evacuation on account of a missed abortion at 9 weeks of gestational. The patient underwent an in vitro fertilization embryo transfer procedure to conceive because she previously had bilateral laparoscopic salpingectomy for the treatment of pregnancy.

The patient was afebrile with stable vital signs. The initial hemoglobin level was $13.1 \mathrm{~g} / \mathrm{dL}$, and the serum beta-human chorionic gonadotropin ( $\beta$-hcg) level was elevated to 799 $\mathrm{mIU} / \mathrm{mL}$. However, the Doppler ultrasonography revealed retained products of conception, $3.1 \times 2.7 \mathrm{~cm}$ in size, showing increased vascularity and prominent vessels situated on the posterior side of the uterine fundus (Fig. 1A and B).

Received: 2018.10.12. Revised: 2018.12.13. Accepted: 2018.12.26. Corresponding author: Ji Hyun Choi

Department of Obstetrics and Gynecology, Chosun University Hospital, Chosun University College of Medicine, 365 Pilmundaero, Dong-gu, Gwangju 61452, Korea

E-mail: themoon6pence@hanmail.net https://orcid.org/0000-0001-7538-6129

Articles published in Obstet Gynecol Sci are open-access, distributed under the terms of the Creative Commons Attribution Non-Commercial License (http://creativecommons. org/licenses/by-nc/3.0/) which permits unrestricted non-commercial use, distribution, and reproduction in any medium, provided the original work is properly cited.

Copyright $\odot 2019$ Korean Society of Obstetrics and Gynecology 


\section{Obstetrics \& Gynecology Science}

Da Joung Shim, et al. Uterine arteriovenous malformation

Spectral flow Doppler showed high velocity flow pattern, and peak systolic velocity within the lesion was above $60 \mathrm{~cm} /$ $\mathrm{s}$ (Fig. 1C). We presumed UAVM accompanied with retained products of conception, based on the medical history and ultrasonographic findings. UAVM could be confirmed on subsequent $\mathrm{CT}$ angiography with $3 \mathrm{D}$ rendering. The $\mathrm{CT}$ scan showed prominent vascularity within the thickened posterior uterine fundus and high enhanced lesion, measuring approximately $65 \mathrm{~mm}$ (Fig. 1D and E). The CT angiography with 3D rendering showed the hypervascular tangles of tortuous vessels (Fig. 1F). Based on the review of prior performed biosy and serial examination, gestational trophoblastic disease (GTD) could be ruled out.

After the diagnosis of UAVM, we consulted an interven-
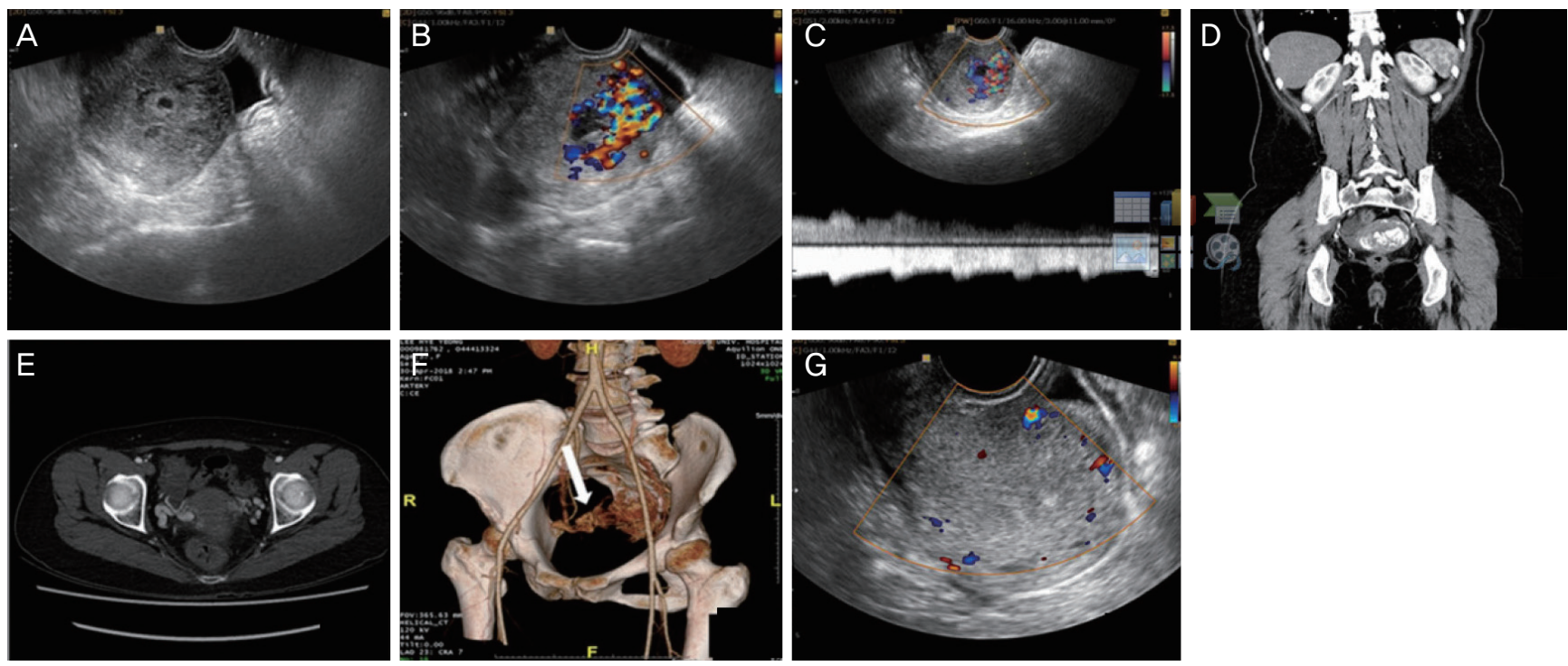

Fig. 1. (A) Transvaginal US shows heterogenous endometrium with an internal echolucent structure. (B) Color Doppler US images reveal multidirectional flow within the posterior wall of the endometrium. (C) Spectral flow Doppler shows high velocity flow pattern (PSV $>60$ $\mathrm{cm} / \mathrm{s}$ ). ( $D, E) C T$ scan shows prominent vascularity within thickened posterior uterine fundus and high enhanced lesion. ( $F$, white arrow) $C T$ angiography with 3D rendering shows the hypervascular tangles of tortuous vessel. (G) After 2 months UAE, follow-up transvaginal US demonstrated complete resolution of UAVM and no vascularity within the lesion. PSV, peak systolic velocity; CT, computed tomography; 3D, 3-dimensional; UAE, uterine artery embolization; UAVM, uterine arteriovenous vascular malformation.
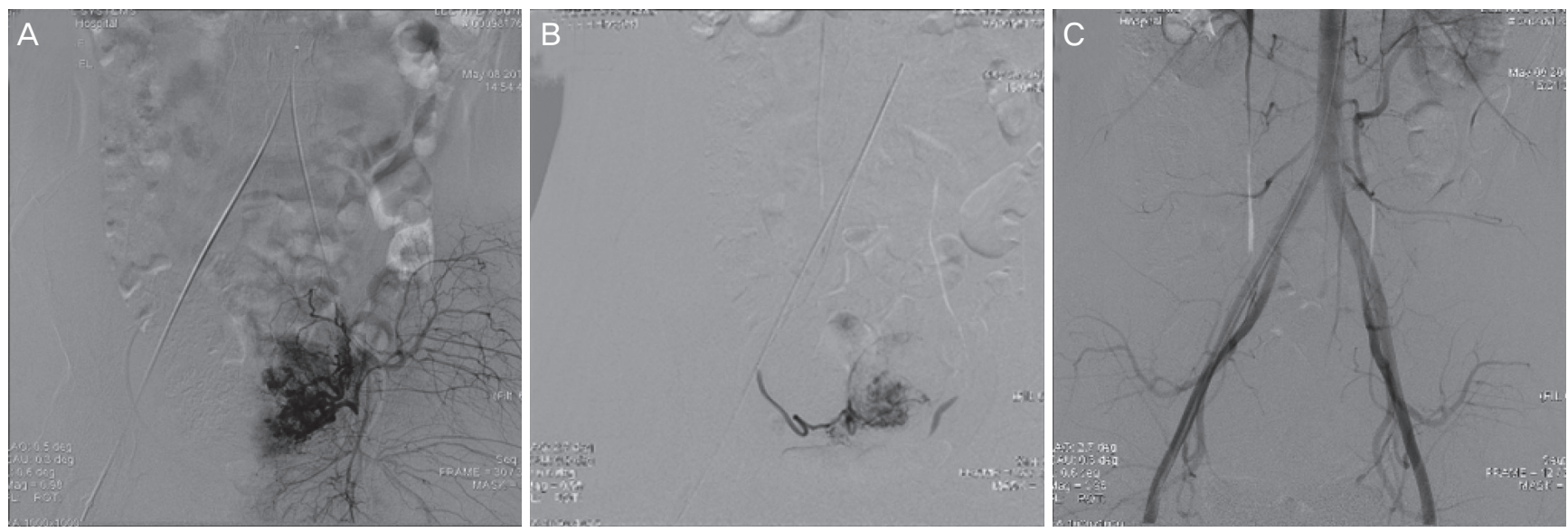

Fig. 2. (A) Left uterine artery. (B) Right uterine artery. Angiography shows UAVM with an early contrast vein filling. (C) Embolization of the AVM was completed. UAVM, uterine arteriovenous vascular malformation; AVM, arteriovenous vascular malformation. 


\section{Obstetrics \& Gynecology Science}

Vol. 62, No. 2, 2019

tional radiologist for the execution of UAE. Pelvic angiography showed bilateral tortuous uterine arteries and marked dilated venous structures with early contrast filling. UAE was performed by an interventional radiologist using Gelfoam particles. Post-uterine embolization angiography confirmed successful occlusion of both uterine arteries (Fig. 2). On the next day, her $\beta$-hcg level was markedly decreased to $88 \mathrm{mlU} /$ $\mathrm{mL}$. On another day, the retained products of conception were safely removed by dilation and curettage, and then the patient was discharged with no procedure-related complications.

Two months later, the patient visited our hospital for her planned follow-up care. During that visit, there was no more vaginal spotting, and the Doppler ultrasonography demonstrated complete resolution of UAVM and no vascularity within the lesion (Fig. 1G). The serum $\beta$-hcg level was normalized, and normal menstruation was resumed. Furthermore, the patient was making thoroughgoing preparations for another pregnancy.

\section{Discussion}

UAVM is a less commonly known disease condition that is potentially life-threatening due to excessive bleeding, and usually results after an invasive procedure such as uterine $\mathrm{Cu}$ rettage or therapeutic abortion [1,2]. UAVM is typically classified as congenital or acquired. The congenital type of UAVM is very rare, and is usually caused by developmental abnormalities of the uterine vessels. The acquired type is more common and may occur due to previous uterine trauma (prior pelvic surgery and curettage), endometrial carcinomas, miscarriage, uterine infections, leiomyomas, endometriosis, intrauterine devices, and GTDs [3]. The acquired type of UAVM is similar to the congenital type in their radiologic findings. Thus, to distinguish between the 2 types, it is important to check the patient's medical history, particularly in women of reproductive age who present with unexplained abnormal vaginal bleeding as anechoic structures as observed on ultrasonography [4]. Even though GTD was a less possible diagnosis, GTD should be considered in the differential diagnosis. If GTD was not ruled out definitively, methotrexate could be applied prophylactically via intramuscular injection [5].

The initial tool for the evaluation of UAVM is mainly ultrasonography. In UAVM, gray-scale ultrasonography shows an observable normal endometrium, whereas the myometrium contains multiple hypo-echoic spaces. In color Doppler ultrasonography, these cystic spaces generate mosaic patterns of color signals representing turbulent flow [4]. Retained placental tissue can lead to hypervascularity with turbulent flow on Doppler investigation as well, leading to confusion with UAVM. Therefore, digital subtraction angiography should be considered as the gold standard of diagnosis $[1,6]$. The preferred diagnostic modalities for diagnosing UAVM also include $C T$ and magnetic resonance imaging.

The management of UAVM varies from observation to total hysterectomy, with the latter being usually considered the best method in case conservative treatments fail [7]. When patients are hemodynamically stable, medical treatment and UAE are the most optimal options $[3,7]$. The current treatment of choice for UAVM is UAE, because it yields a high success rate associated with only few complications compared to other methods and provides a fertilitypreserving potential, because UAVM is generally diagnosed among women of childbearing age. However, hysterectomy can be the treatment of choice in post-menopausal women or as an emergency treatment in life-threatening situations [8-10], and the use of gonadotropin-releasing hormone agonists can be attempted to treat UAVM in stable patients [11]. In mildly symptomatic patients, spontaneous resolution of UAVM can be expected. That is why close clinical followup and radiologic investigations are required for decisions of definitive treatment. As demonstrated here, if reproductiveage women show repeated abnormal vaginal bleeding after dilatation and curettage, UAVM could also be considered as the reason.

\section{Conflict of interest}

No potential conflict of interest relevant to this article was reported.

\section{Patient consent}

The patients provided written informed consent for the publication and the use of their images. 


\section{Obstetrics \& Gynecology Science}

Da Joung Shim, et al. Uterine arteriovenous malformation

\section{References}

1. Grivell RM, Reid KM, Mellor A. Uterine arteriovenous malformations: a review of the current literature. Obstet Gynecol Surv 2005;60:761-7.

2. Ahn HY, Park IY, Lee G, Kim SJ, Shin JC. Uterine arteriovenous malformation. Arch Gynecol Obstet 2005;271:172-5.

3. Oguz Y, Gonca Eldem F, Cil B, Sanhal C, GençosmanoğluTürkmen $G$, Aykan $Y$, et al. Uterine arteriovenous malformation. Gynecol Obstet Reprod Med 2018 Jul [Epub]. https://doi.org/10.21613/GORM.2018.776.

4. Polat P, Suma S, Kantarcý M, Alper F, Levent A. Color Doppler US in the evaluation of uterine vascular abnormalities. Radiographics 2002;22:47-53.

5. Kim SM, Ahn HY, Choi MJ, Kang YD, Park JW, Park CH, et al. Uterine arteriovenous malformation with positive serum beta-human chorionic gonadotropin: embolization of both uterine arteries and extra-uterine feeding arteries. Obstet Gynecol Sci 2016;59:554-8.
6. Müngen E. Vascular abnormalities of the uterus: have we recently over-diagnosed them? Ultrasound Obstet Gynecol 2003;21:529-31.

7. Rosa e Silva JC, de Aguiar FM, de Sa Rosa e Silva AC, Candido dos Reis FJ, Poli Neto OB, Nogueira AA. Conservative management of large uterine arteriovenous malformation: case report. Pol J Radiol 2015;19:202-5.

8. Singh N, Tripathi R, Mala YM, Tyagi S, Tyagi S, Singh C. Varied presentation of uterine arteriovenous malformations and their management by uterine artery embolisation. J Obstet Gynaecol 2014;34:104-6.

9. Delotte J, Chevallier P, Benoit B, Castillon JM, Bongain A. Pregnancy after embolization therapy for uterine arteriovenous malformation. Fertil Steril 2006;85:228.

10. Hashim H, Nawawi O. Uterine arteriovenous malformation. Malays J Med Sci 2013;20:76-80.

11. Nonaka T, Yahata T, Kashima K, Tanaka K. Resolution of uterine arteriovenous malformation and successful pregnancy after treatment with a gonadotropin-releasing hormone agonist. Obstet Gynecol 2011;117:452-5. 